\title{
An analysis on the adjuvant efficacy of intravenous or perineural administered dexamethasone in erector spina plane block applied to patients who had thoracotomy: a prospective randomized double-blind study
}

\author{
๑1)Tuna Ertürk, ๑Bülent Barış Güven, @Ayşın Ersoy \\ University of Health Sciences, Sultan 2. Abdulhamid Han Training And Research Hospital, Department of Anesthesiology, İstanbul, Turkey
}

Cite this article as: Ertürk T, Güven BB, Ersoy A. An analysis on the adjuvant efficacy of intravenous or perineural administered dexamethasone in erector spina plane block applied to patients who had thoracotomy: a prospective randomized double-blind study. Anatolian Curr Med J 2022; 4(1); 13-18.

\begin{abstract}
Aim: Thoracic surgery is one of the surgical procedures that necessitates effective pain management both before and after the procedure. Because of the adverse effects of opioids in thoracic surgeries, regional anesthesia has been widely used for postoperative analgesia. One of the most recent techniques used with this indication is erector spina plane block (ESPB). Many studies in regional anesthesia have shown that adding dexamethasone to local anesthetics as an adjuvant agent prolongs the analgesic effect. The purpose of the present study was to assess the effect of dexamethasone, which is used as a perineural and intravenous adjuvant, on the duration and level of analgesia of ESPB used in thoracic surgeries.

Material and Method: This prospective, randomized controlled study included 60 patients who would have a thoracotomy under general anesthesia and ESP for peri-operative analgesia. Patients were divided into three groups: those who received perineural dexamethasone (Group $\mathrm{PN}$ ), those who received IV dexamethasone (Group IV), and those who did not receive any dexamethasone at all (Group C-Control). Numerical rating scale (NRS) scores, rescue analgesic drug use and block times were recorded at the postoperative $2^{\text {nd }}, 6^{\text {th }}, 12^{\text {th }}, 24^{\text {th }}$ and $36^{\text {th }}$ hours.

Results: There was no statistically significant $(\mathrm{p}>0.05)$ difference in blocking times or additional analgesic use between the three groups. There was no statistically significant ( $>0.05$ ) difference in the NRS scores when the patients' pain levels were measured in all three groups. 36th hour NRS scores and additional analgesic use were higher in the group that did not receive IV or perineural dexamethasone.

Conclusion: NRS scores and rescue analgesic use were similar between ESPB groups with intravenous or perineural addition of 4 mg dexamethasone and ESPB group administered without dexamethasone. However, the NRS score average in the group C was greater than 3, particularly in the $36^{\text {th }}$ hour NRS data, whereas the NRS scores in the group PN and group IV were less than 2.
\end{abstract}

Keywords: Erector spina plane block, perineural dexamethasone, intravenous dexamethasone, thoracotomy, postoperative analgesia

\section{INTRODUCTION}

Thoracic surgeries, whether open or closed (video assisted), are among the most painful surgical procedures and necessitate effective analgesia (1). The pain that develops as a result of insufficient analgesia prevents the patient from breathing deeply and coughing, resulting in respiratory distress such as atelectasis, hypoxia, and pneumonia (2).

Opioids used as traditional analgesics have the potential for a number of unwanted side effects (such as nausea, vomiting, constipation, sedation, urinary retention, and pruritus), tolerance, and dependence. Regional anesthesia and peripheral blocks, which are commonly used in thoracic surgeries, are more effective at controlling postoperative pain than other analgesia strategies and they also reduce the use of opioid drugs (3).
Thoracic epidural analgesia (TEA) and thoracic paravertebral block (TPVB) are the most commonly used pain management techniques following thoracic surgery. They are, however, technically challenging to implement and have a high failure rate (up to 15 percent in TEA). Furthermore, both methods have significant risks, including pneumothorax, dural puncture, hematoma, infection, and nerve injury $(4,5)$. In recent years, facial plane blocks have brought benefits such as ease of application, effective analgesia, and a low complication rate. The most recent of these methods is the erector spina plan block (ESPB), which was described in 2016 by Forero et al. (6).

Many researchers have been looking for the best analgesic adjuvant that both extends the duration of analgesia and 
has fewer side effects after peripheral nerve block. Even though many agents failed this test (opioids, ketamine, clonidine, etc.), it has been demonstrated in some studies that the addition of dexamethasone to local anesthetics, both perineural and intravenously, prolongs the analgesic effect $(7,8)$.

There are studies in the literature regarding the adjuvant efficacy of dexamethasone, which is used perineural or intravenously. In our study, we aimed to evaluate the effect of the adjuvant efficacy of dexamethasone on the level and duration of analgesia in ESPB, which has been defined recently in thoracic surgeries.

\section{MATERIAL AND METHOD}

This prospective, randomized controlled and doubleblind study was carried out with the permission of Health Sciences University Clinical Researches Ethics Committee (Date: 03.12.2020, Decision No: 20-110). Informed consent was obtained from all patients. All procedures were carried out in accordance with the ethical rules and the principles of the Declaration of Helsinki. The Consolidated Standards of Reporting Trials flow diagram was used for enrollment and allocation of patients (Figure 1).

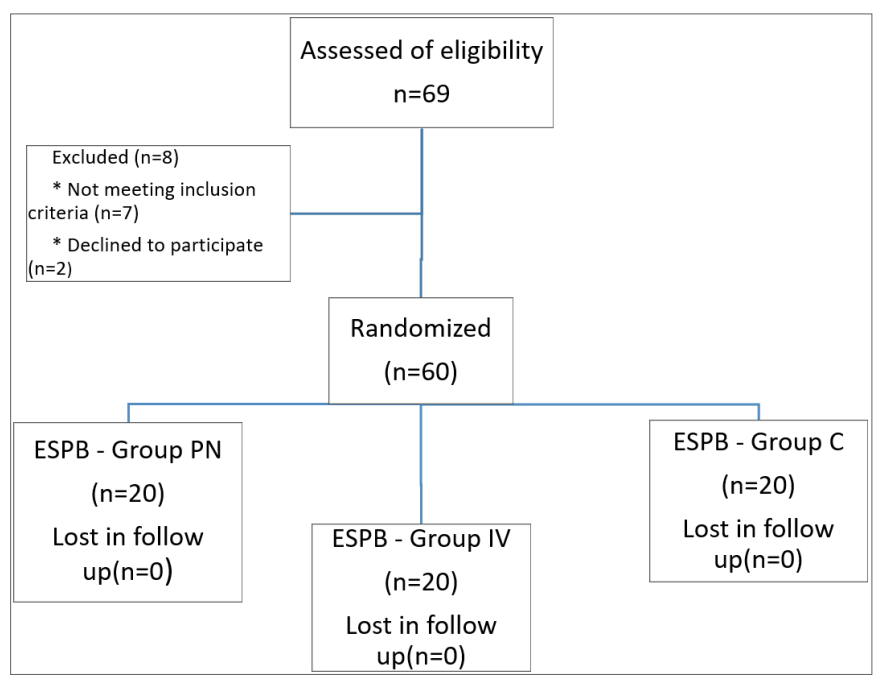

Figure 1. Study flowchart. ESPB, erector spinae plane block. PN: Perineural, IV: Intravenous, C: Control

In the present study, sixty ASA I-III patients, aged 1870 years, who will undergo thoracotomy surgery under general anesthesia and are scheduled to receive ESP for peri-postoperative analgesia, were included. Patients in the study were divided into three groups: those who received perineural dexamethasone (Group PN), those who received IV dexamethasone (Group IV), and those who did not receive any dexamethasone at all (Group CControl).
Patients aged younger than 18 years, older than 70 years, with ASA physical status classification IV, infection of the skin at the site of needle puncture area, with known allergies to any of the study drugs, receiving antithrombotic treatment and whose coagulation parameters are abnormal were excluded from the study.

In the premedication room, patients were informed about ESPB. Data on demographics were collected. Each patient was sedated with $0.03 \mathrm{mg} \mathrm{kg}-1$ midazolam. In the operating room all patients had received standardized monitoring which included $\mathrm{SpO}_{2}$, ECG, noninvasive blood pressure monitoring.

The patient, whose hemodynamics were deemed stable, was placed in a sitting position. Following sterilization of the procedure area, the USG (SonoSite M-Turbo HFL50x/15-6 MHz Lineer Transducer SonoSite, Inc. Bothell, WA 98021 USA) probe was dressed sterile with a camera sheath. A $4 \mathrm{~cm}$ depth-adjusted linear probe was placed in the parasagittal plane, about $3 \mathrm{~cm}$ lateral to the T5 spinous process (axillary fold level), under USG guidance. After observing the T5 transverse process with an in-plane approach, an $80 \mathrm{~mm}$ long block needle (Stimuplex B. Braun R) was craniocaudally inserted through the skin. When the trapezius, rhomboid, and erector spina muscles were passed and the needle rested on the transverse process (about $3 \mathrm{~cm}$ in depth), $1 \mathrm{~mL}$ of 0.9 percent $\mathrm{NaCl}$ test dose was administered between the erector spina muscle fascia and the vertebral transverse process, the muscle fascia was opened, and the needle location was confirmed (Picture 1).

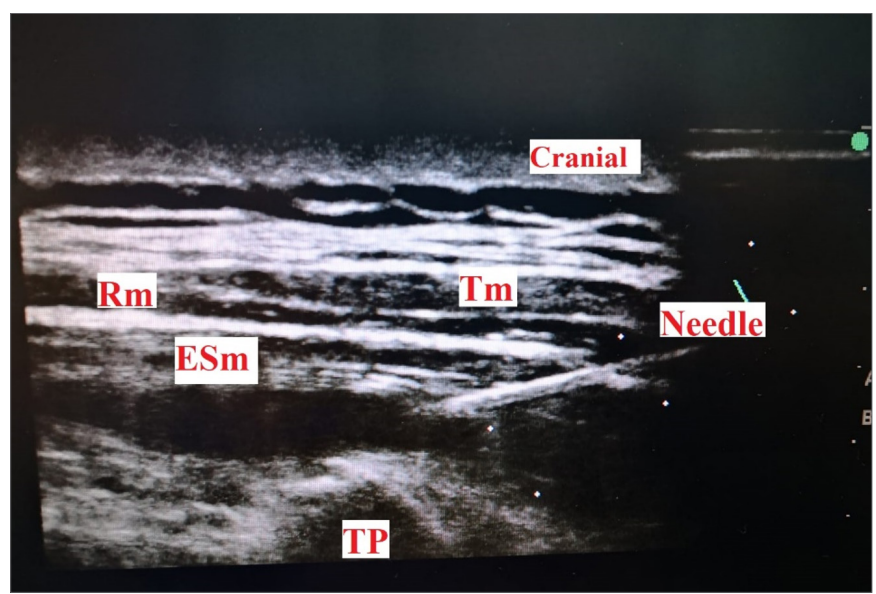

Picture 1. Ultrasonographic image and anatomical landmarks

In group C, $30 \mathrm{~mL}$ of 0.25 percent bupivacaine was injected into the erector spina plane, followed by ESPB. In the group $\mathrm{PN}$, the erector spina plan was given $30 \mathrm{ml}$ of 0.25 percent bupivacaine and $4 \mathrm{mg}$ of dexamethasone, and ESPB was used. In the group IV, $30 \mathrm{~mL}$ of 0.25 percent bupivacaine was injected into the erector spina plane and ESPB, and $4 \mathrm{mg}$ of dexamethasone was injected intravenously. All blockages were performed by the same anesthesiologist 
who was in charge of the thoracic surgery room. The study was conducted in a double-blind fashion, with neither the patient nor the anesthesia team, who administered general anesthesia and followed up on the patient, knowing which group the patient belonged to.

Following the blockade, the patient was placed in the supine position and intubated after anesthesia induction (intravenous propofol $2 \mathrm{mg} / \mathrm{kg}$, rocuronium $0.5 \mathrm{mg} / \mathrm{kg}$, fentanyl $1 \mathrm{mcg} / \mathrm{kg}$ ). In addition, maintenance anesthesia (50-50 percent $\mathrm{O}_{2}$ - air and $2 \%$ sevoflurane) was planned by connecting to a mechanical ventilator.

At the end of the surgery, all of the patients received paracetamol $1 \mathrm{~g}$ IV and petidin (Meperidin, Dolantin) 30 $\mathrm{mg}$ as a standard for postoperative analgesia. For preventing postoperative nausea and vomiting ondansetron $(8 \mathrm{mg}$ IM) was also administered to all patients.

After being awakened at the end of the procedure, the patients were transferred to the post-operative care unit. Patients with an Aldrete score of 9-10 were transferred to their wards after connecting an intravenous tramadol patient-controlled analgesia device $(5 \mathrm{mg} / \mathrm{ml}$ concentration, $20 \mathrm{mg}$ bolus dose, 30 -minute lock-in time).

Numerical rating scale (NRS) scores were recorded at 2-6-12, 24 and 36 hours after surgery, as well as additional analgesic medication and administration time if necessary. After the first evaluation hour with NRS $>4$, patients in both groups were started on IV analgesia treatment (paracetamol 1 gr IV 3x1, dexketoprofen 50 $\mathrm{mg}$ IV as an additional analgesic). If the VAS was 4 and the patient did not require analgesics, the paracetamol dose was skipped.

\section{Statistical Analysis}

Mean, standard deviation, median, minimum, maximum, frequency, and ratio values were used in the descriptive statistics of the data. The Kolmogorov-Smirnov test was used to determine variable distribution. In the analysis of quantitative independent data, ANOVA and Kruskalwallis were employed. The Chi-square test was used to analyze qualitative independent data. The analysis was carried out using the SPSS 27.0 program. The results were evaluated at the $95 \%$ confidence interval and the significance level at $\mathrm{p}<0.05$.

The calculation for the strength of the present study was performed on the page https://www. dssresearch.com/KnowledgeCenter/toolkitcalculators/ samplesizecalculators.aspx by referring to the " Dexamethasone as a local anesthetic adjuvant in bilateral ultrasound guided erector spinae plane block can provide a long-lasting analgesia in laparotomic abdominal surgery. Fusco P, et al. Minerva Anestesiol. 2019; 85:1144-5." study. A power analysis was carried out, assuming Type I error $=0.05$ and Type II error $=0.2$ ( $80 \%$ power to detect this difference), then 20 patients were required in each group.

Randomization was performed according to computergenerated random number tables and allocation to treatment group was done using the sealed opaque envelope technique.

\section{RESULTS}

The study included 60 patients, 30 of whom were women and 30 of whom were men. When demographic information, BMI values, and ASA classification distributions were compared, there was no statistically significant difference between the groups $\mathrm{C}, \mathrm{PN}$, and IV $(\mathrm{p}>0.05)$ (Table 1).

\begin{tabular}{|c|c|c|c|c|}
\hline & $\begin{array}{c}\text { Group PN } \\
\text { n \% Median }\end{array}$ & $\begin{array}{c}\text { Group IV } \\
\text { n \% Median }\end{array}$ & $\begin{array}{c}\text { Group C } \\
\text { n \% Median }\end{array}$ & $\mathbf{p}$ \\
\hline Age & 62.0 & 58.0 & 56.5 & $0.456 \mathrm{~A}$ \\
\hline Sex & & & & $0.449 X^{2}$ \\
\hline Female & $1260.0 \%$ & $840.0 \%$ & $1050.0 \%$ & \\
\hline Malea & $840.0 \%$ & $1260.0 \%$ & $1050.0 \%$ & \\
\hline Height & 163.5 & 167.5 & 168.5 & $0.610 \mathrm{~A}$ \\
\hline Weight & 73.0 & 72.5 & 75.0 & $0.793 \mathrm{~A}$ \\
\hline BMI & 26.1 & 25.2 & 26.0 & $0.704 \mathrm{~A}$ \\
\hline ASA & & & & $0.676 \mathrm{X}^{2}$ \\
\hline I & $15.0 \%$ & $315.0 \%$ & $420.0 \%$ & \\
\hline II & $1680.0 \%$ & $1365.0 \%$ & $1470.0 \%$ & \\
\hline III & $315.0 \%$ & $420.0 \%$ & $210.0 \%$ & \\
\hline
\end{tabular}

There was no statistically significant $(\mathrm{p}>0.05)$ difference in blocking times or rescue analgesic use between the three groups (Table 2). Although NRS scores and rescue analgesic use at $36^{\text {th }}$ hour were not statistically significant, they were lower in favor of PN and IV groups (Graph 1). Especially in 36th hour NRS data, NRS score average was above 3 in Group C, NRS scores were around 2 and similar in PN and IV groups.

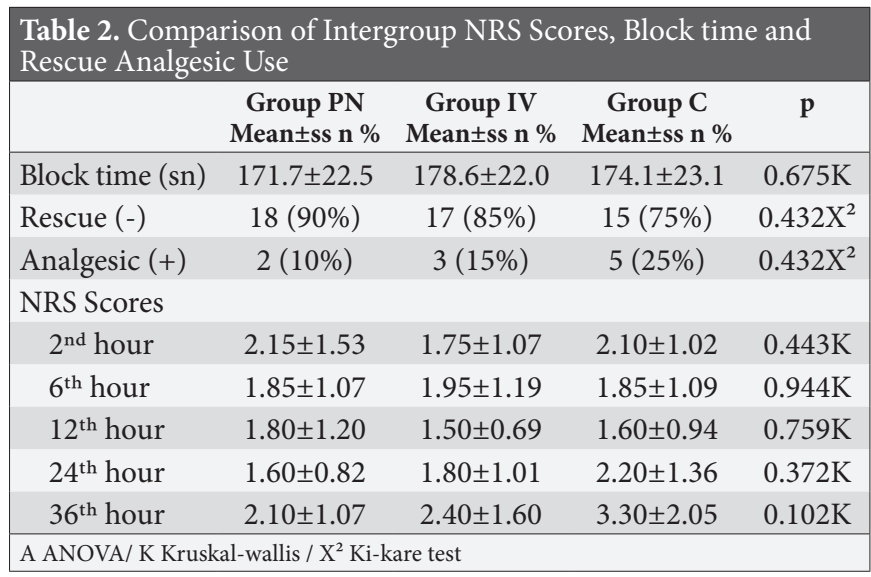


There was no statistically significant $(\mathrm{p}>0.05)$ difference in the NRS scores when the patients' pain levels were measured in all three groups. NRS scores were similar at the $2^{\text {nd }}, 6^{\text {th }}, 12^{\text {th }}, 24^{\text {th }}$, and $36^{\text {th }}$ hours (Table 2) (Figure 2).

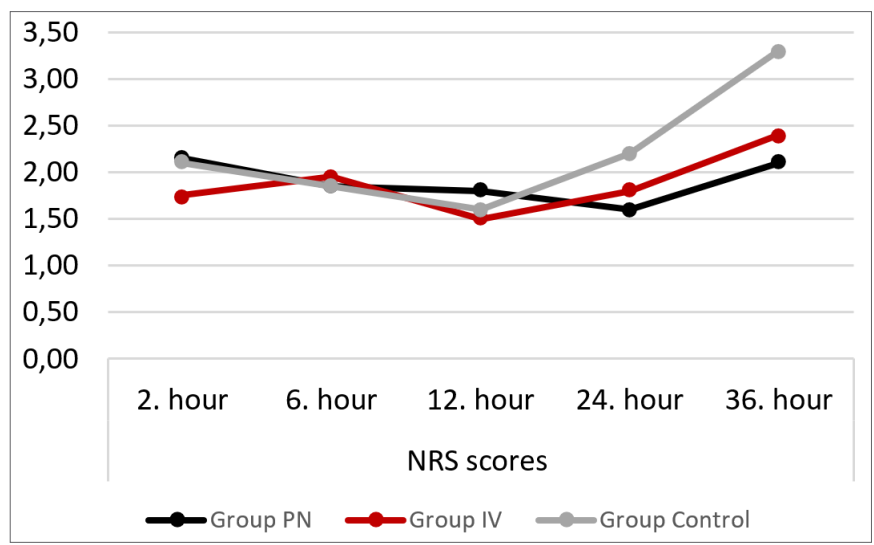

Figure 2. NRS scores and timeline

\section{DISCUSSION}

The objective of the present study was to assess the effect of dexamethasone administered intravenously or perineurally as an adjuvant in ultrasound guided ESPB in patients undergoing thoracotomy surgery. There was no statistically significant difference in NRS scores, or additional analgesic need between the ESP block groups that received $4 \mathrm{mg}$ dexamethasone intravenously (IV) or perineurally $(\mathrm{PN})$ versus those that did not receive dexamethasone (C). However, while the NRS score average in the group $\mathrm{C}$ was above 3, particularly in the 36th hour NRS data, the NRS scores in the groups PN and IV were around 2 and similar.

Effective pain management following thoracic surgery is important because it has a greater impact on mortality and morbidity than other major surgeries by reducing the stress response and serious pulmonary complications that might occur as a result of changes in lung functions following thoracic surgery $(9,10)$.

The authors recommend a multimodal analgesia approach that includes regional and systemic analgesia techniques for pain that develops after a thoracotomy $(11,12)$. Different regional anesthesia techniques are used in thoracic surgery, the most common of which are TEA, TPVB, and infraclavicular block. Nevertheless, because of difficulties in application, susceptibility to complications, and failed attempts, these methods might not be preferred for postoperative analgesia $(4,5,13)$. As a result, ESPB, which is simple to administer under ultrasound guidance, has a low complication rate, and whose efficacy has been demonstrated in numerous studies, was used on the patients in the present study $(14,15)$.
Ultrasound-guided ESPB is an interfacial plane block developed in 2016 by Forero et al. (6) to treat thoracic neuropathic pain. Even though it was initially defined for use in chronic pain, it has since been used as a postoperative analgesia method in a variety of surgical procedures ranging from shoulder to hip (16). Many studies have been conducted to investigate the postoperative analgesic efficacy of ESPB in thoracic surgeries. It has been agreed that it provides effective postoperative analgesia and reduces complications such as atelectasis by allowing the patient to breathe comfortably and cough without pain $(6,14,15)$.

Many adjuvants, including neostigmine, tramadol, or clonidine, have been investigated for their ability to extend the duration of action of local anesthetics used in regional anesthesia, but negative results have been obtained (17). Dexamethasone was first used perineurally 12 years ago, and it has since been the subject of numerous clinical studies. The mechanism by which dexamethasone prolongs block time is not completely understood. Mechanisms proposed include reduced nociceptive $\mathrm{C}$-fiber activity via glucocorticoid receptors, an effect via inhibitory potassium channels, a local vasoconstrictive effect, and a systemic anti-inflammatory effect $(18,19)$. Some studies have been conducted on the adjuvant effect of dexamethasone, which is used intravenously at moderate doses, due to its systemic anti-inflammatory effect.

Crystallization might occur during in vitro studies when ropivacaine, rather than bupivacaine, is combined with dexamethasone due to the high $\mathrm{pH}$ of dexamethasone and the incompatibility of ropivacaine with alkaline solutions (20). Furthermore, systemic dexamethasone administration in moderate doses is a recommended option for effective postoperative analgesia, regardless of whether it is associated with peripheral nerve block (21). Based on this data, bupivacaine and moderate doses of dexamethasone were chosen as local anesthetics in ESPB applications of the present study.

According to a recent meta-analysis, combining perineural dexamethasone with long-acting local anesthetics increases the duration of analgesia by 8 hours when compared to placebo (22).

In their randomized controlled study, Desmet et al. (23) investigated both routes of administration and discovered that the duration of analgesia they produced was comparable $(24,25)$. In a meta-analysis involving 914 patients and 11 randomized controlled trials, Baeriswyl M.et al. (18) found that perineural dexamethasone slightly prolonged the duration of analgesia without causing any side effects. The duration of analgesia increased by 17 percent when dexamethasone was injected perineurally (mean 3.0 hours) compared to systemic administration, according to the same study. 
While some studies concluded that perineural dexamethasone was superior in terms of analgesia, others discovered statistically significant but clinically insignificant differences (26-28). In the present study, NRS scores up to the $24^{\text {th }}$ hour and the use of additional analgesics were comparable in all three groups; however, the $36^{\text {th }}$-hour NRS scores were lower in the groups PN and IV, but this difference was not statistically significant.

The majority of ESPB research focuses on the analgesic effects in the first 24 hours after surgery (29-32). In addition to the adjuvant effect of dexamethasone, its analgesic effect at 36 hours was evaluated in the present study. Even though the NRS scores and use of additional analgesics were comparable up to the $24^{\text {th }}$ hour, the mean of the 36 th hour NRS scores was higher in the group $\mathrm{C}$ that did not use dexamethasone. During the $36^{\text {th }}$-hour evaluation, additional analgesics were given to 5 patients in the group $\mathrm{C}$ who did not receive dexamethasone, serious pain was observed in two patients, and the NRS score was 7 in one patient and 8 in the other.

The results of the present study can be evaluated within some limitations. According to previous research, the adjuvant effects of low-dose (4 mg) and high-dose (8$10 \mathrm{mg}$ ) dexamethasone, administered perineurally or intravenously, were also comparable. Furthermore, by controlling the NRS and additional analgesic times every hour and/or by increasing the number of cases, more valuable information can be obtained about whether dexamethasone used as an adjuvant prolongs the duration of analgesic effect.

\section{CONCLUSION}

In the present study, all three groups had similar mean NRS scores (Group PN:1.6, Group IV:1.6, Group C:1.9) and rescue analgesic use (Group PN:2/20, Group IV:3/20, Group C:5/20 ) in the first 24 hours. ESPB blockade provided effective and adequate analgesia to patients in all three groups.

However, when the $36^{\text {th }}$-hour NRS scores and additional analgesic use were considered, lower mean \pm ss NRS scores and less rescue analgesic use were found in the group PN $(2.10 \pm 1.07)$ and group IV $(2.40 \pm 1.60)$ compared to the group C $(3.30 \pm 2.05)$, but this was not statistically significant.

\section{ETHICAL DECLARATIONS}

Ethics Committee Approval: The study was carried out with the permission of Health Sciences University Clinical Researches Ethics Committee (Date: 03.12.2020, Decision No: 20-110).
Informed Consent: All patients signed the free and informed consent form.

Referee Evaluation Process: Externally peer-reviewed.

Conflict of Interest Statement: The authors have no conflicts of interest to declare.

Financial Disclosure: The authors declared that this study has received no financial support.

Author Contributions: All of the authors declare that they have all participated in the design, execution, and analysis of the paper, and that they have approved the final version.

\section{REFERENCES}

1. Homma T, Doki Y, Yamamoto Y, et al. Risk factors of neuropathic pain after thoracic surgery. J Thorac Dis 2018; 10: 2898-907.

2. Xie C, Ran G, Chen D, Lu Y. A narrative review of ultrasoundguided serratus anterior plane block. Ann Palliat Med 2021; 10: 700-6.

3. Pirsaharkhiz N, Comolli K, Fujiwara W, et al. Utility of erector spinae plane block in thoracic surgery. J Cardiothorac Surg 2020; 15: 1-6.

4. Romero A, Garcia JEL, Joshi GP. The state of the art in preventing postthoracotomy pain. Semin Thorac Cardiovasc Surg 2013; 25: 116-24.

5. Moustafa MA, Alabd AS, Ahmed AMM, Deghidy EA. Erector spinae versus paravertebral plane blocks in modified radical mastectomy: Randomised comparative study of the technique success rate among novice anaesthesiologists. Indian J Anaesth 2020; 64: 49-54.

6. Forero M, Adhikary SD, Lopez H, Tsui C, Chin KJ. The erector Spinae plane block: a novel analgesic technique in thoracic neuropathic pain. Reg Anesth Pain Med 2016; 41: 621-7.

7. Hewson D, Bedforth N, McCartney C, Hardman J. Dexamethasone and peripheral nerve blocks: back to basic (science). Br J Anaesth 2019; 122: 411-2.

8. Mchardy PG, Singer O, Awad IT, et al. Comparison of the effects of perineural or intravenous dexamethasone on low volume interscalene brachial plexus block: a randomised equivalence trial. Br J Anaesth 2020; 124: 84-91.

9. Goto T. What is the best pain control after thoracic surgery? J Thorac Dis 2018; 10: 1335-8.

10. Cassi LC, Biffoli F, Francesconi D, Petrella G, Buonomo O. Anesthesia and analgesia in breast surgery: the benefits of peripheral nerve block. Eur Rev Med Pharmacol Sci 2017; 21: 1341-5.

11. Szewczyk J, Nguyen BH, Villamizar N, Nguyen DM. Achieving pain control with opioid-sparing multimodal analgesic strategydoing more with less by enhanced recovery after thoracic surgery protocol: A Review. J Anesthesiol Pain Ther 2020, 1.3.

12. Wenk M, Schug SA. Perioperative pain management after thoracotomy. Curr Opin Anaesthesiol 2011; 24: 8-12.

13. Niesen AD, Jacob AK, Law LA, Sviggum HP, Johnson RL. Reg Anesth Pain Med 2020; 45: 813-7.

14. Seelam S, Nair AS, Christopher, Upputuri O, Naik V, Rayani BK. Efficacy of single-shot ultrasound-guided erector spinae plane block for postoperative analgesia after mastectomy: a randomized controlled study. Saudi J Anaesth 2020; 14: 22-7.

15. Gürkan Y, Aksu C, Kus A, Yörükoğlu UH. Erector spinae plane block and thoracic paravertebral block for breast surgery compared to IV-morphine: a randomized controlled trial. J Clin Anesth 2020; 59: 84-8. 
16. Peker K, Peker SA. The effect of erector spinae plane block on cost of percutaneous nephrolithotomy surgery. J Health Sci Med 2020; 3: 409-14

17. Axelsson K, Gupta A. Local anaesthetic adjuvants: neuraxial versus peripheral nerve block. Curr Opin Anaesthesiol 2009; 22: $649-54$.

18. Baeriswyl M, Kirkham KR, Jacot-Guillarmod A, Albrecht E. Efficacy of perineural vs systemic dexamethasone to prolong analgesia after peripheral nerve block: a systematic review and meta-analysis. Br J Anaesth 2017; 1; 119: 183-91.

19. Oğurel T, Örnek K, Oğurel R, Onaran Z. The effect of intracameral dexamethasone used at the end of cataract surgery on macular edema in eyes with diabetic retinopathy. J Health Sci Med 2019; 2: $18-23$

20. Watkins TW, Dupre S, Coucher JR. Ropivacaine and dexamethasone: a potentially dangerous combination for therapeutic pain injections. J Med Imaging Radiat Oncol 2015; 59: $571-7$

21. Desmet M, Vanneste B, Reynvoet $M$, et al. A randomised controlled trial of intravenous dexamethasone combined with interscalene brachial plexus blockade for shoulder surgery. Anaesthesia 2015; 70: 1180-5.

22. Albrecht E, Kern C, Kirkham KR. Perineural vs intravenous administration of dexamethasone: more data are available. Br J Anaesth 2015; 114: 160

23. Desmet M, Braems H, Reynvoet M, et al. I.V. and perineural dexamethasone are equivalent in increasing the analgesic duration of a single-shot interscalene block with ropivacaine for shoulder surgery: a prospective, randomized, placebocontrolled study. Br J Anaesth 2013; 111: 445-52.

24. Kawanishi R, Yamamoto K, Tobetto Y, et al. Perineural but not systemic low-dose dexamethasone prolongs the duration of interscalene block with ropivacaine: a prospective randomized trial. Local Reg Anesth 2014; 7: 5-9.

25. Leurcharusmee P, Aliste J, Van Zundert TC, et al. A multicenter randomized comparison between intravenous and perineural dexamethasone for ultrasound-guided infraclavicular block. Reg Anesth Pain Med 2016; 41: 328-33.

26. Aliste J, Leurcharusmee P, Engsusophon P, et al. A randomized comparison between intravenous and perineural dexamethasone for ultrasound-guided axillary block. Can J Anaesth 2017; 64: 2936.

27. Holland D, Amadeo RJJ, Wolfe S, et al. Effect of dexamethasone dose and route on the duration of interscalene brachial plexus block for outpatient arthroscopic shoulder surgery: a randomized controlled trial. Can J Anaesth 2018; 65: 34-45.

28. Kahn RL, Cheng J, Gadulov Y, Fields KG, YaDeau JT, Gulotta LV. Perineural low-dose dexamethasone prolongs interscalene block analgesia with bupivacaine compared with systemic dexamethasone: a randomized trial. Reg Anesth Pain Med 2018; 43: 572-9.

29. Fu J, Zhang G, Qiu Y. Erector spinae plane block for postoperative pain and recovery in hepatectomy: A randomized controlled trial. Medicine 2020; 99: e22251.

30. Mostafa SF, Abdelghany MS, Abu Elyazed MM. Ultrasoundguided erector spinae plane block in patients undergoing laparoscopic bariatric surgery: a prospective randomized controlled trial. Pain Pract 2021; 21: 445-53.

31. Luo R, Tong X, Yan W, Liu H, Yang L, Zuo Y. Effects of erector spinae plane block on postoperative pain in children undergoing surgery: A systematic review and meta-analysis of randomized controlled trials. Pediatric Anesthesia, 2021, 31: 1046-55.
32. Herman JA, Urits I, Kaye AD, Urman RD, Viswanath $\mathrm{O}$. Erector Spinae Plane Block (ESPB) or Quadratus Lumborum Block (QLB-II) for laparoscopic cholecystectomy: Impact on postoperative analgesia. J Clin Anesth 2020; 66: 109958. 